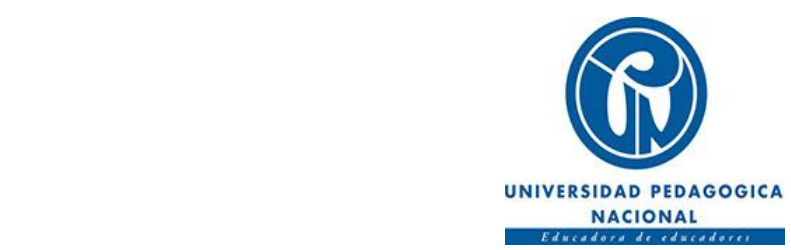

LENGUAJES DE PAZ: UNA CONSTRUCCIÓN SOCIAL FEMENINA UNA EXPERIENCIA DE LA INICIATIVA LOCAL DE PAZ DE FACATATIVÁ CUNDINAMARCA

\author{
Autora \\ Zolángela Enríquez Duque
}

Tutora

Andrea Mireya Jiménez Pinzón

UNIVERSIDAD PEDAGÓGICA NACIONAL

MAESTRÍA EN DESARROLLO EDUCATIVO Y SOCIAL

BOGOTÁ D,C.

2016 


\begin{tabular}{|c|c|}
\hline \multicolumn{2}{|c|}{ FORMATO } \\
\hline \multicolumn{2}{|c|}{ RESUMEN ANALÍTICO EN EDUCACIÓN - RAE } \\
\hline Código: FOR020GIB & Versión: 01 \\
\hline Fecha de Aprobación: 10-10-2012 & Página 2 de 30 \\
\hline
\end{tabular}

\section{Información General}

\begin{tabular}{||l|l||}
\hline Tipo de documento & Tesis de grado de Maestría \\
\hline Acceso al documento & Universidad Pedagógica Nacional. Biblioteca Central. \\
\hline Titulo del documento & $\begin{array}{l}\text { Lenguajes de paz: una construcción social femenina. Una experiencia de } \\
\text { la iniciativa local de paz de Facatativá - Cundinamarca. }\end{array}$ \\
\hline Autor(es) & Enríquez Duque, Zolangela. \\
\hline Director & Jiménez Pinzón, Andrea. \\
\hline Publicación & Bogotá, Universidad Pedagógica Nacional, 2015, XX p. \\
\hline Unidad Patrocinante & Fundación Centro Internacional de Educación y Desarrollo Humano CINDE \\
& \\
\hline Palabras Claves & $\begin{array}{l}\text { Desarrollo Humano, Construccionismo Social, Prácticas, Subjetividad } \\
\text { Femenina, Lenguajes de Paz. }\end{array}$ \\
\hline
\end{tabular}

\section{Descripción}

Tesis de grado donde la autora presenta los resultados parciales de la sistematización de experiencia de la Iniciativa Local de Paz - ILP, denominada Mujeres Activas que se desarrolla en Facatativá Cundinamarca, en convenio con el Secretariado Nacional de Pastoral Social - SNPS y Ficonpaz. Dicha iniciativa se estudia desde dos puntos de comprensión.

El primero hace alusión a las prácticas que promueven las mujeres a través de sus procesos de construcción como agrupación. Y el segundo, sobre los sentidos que se establecen alrededor de sus prácticas comunicativas en la manera como configuran lenguajes de paz.

Es importante precisar que los resultados expuestos aquí, aluden tanto a prácticas asociadas con actividades de la Iglesia, como con acciones de carácter social y económico emprendidas por las mujeres, y que dan cuenta de su participación en la iniciativa, en las cuales hacen uso del lenguaje como elemento instituyente para la construcción de paz. 


\begin{tabular}{|c|c|}
\hline \multicolumn{2}{|c|}{ FORMATO } \\
\hline \multicolumn{2}{|c|}{ RESUMEN ANALITICO EN EDUCACIÓN - RAE } \\
\hline \multicolumn{2}{|c|}{ Código: FOR020GIB } \\
\hline Fecha de Aprobación: 10-10-2012 & Persión: 01 \\
\hline
\end{tabular}

\section{Fuentes}

Arendt, H. (1997) ¿Qué es la política?, trad. de Rosa Sala Carbón, Barcelona, España. Paidós. Edición póstuma 1993.

Berger, P, \& Luckmann, T. (1996). La construcción social de la realidad. Madrid, España Editorial Pingüino.

CINDE, Universidad de Manizales, Universidad Pedagógica (26 de Agosto de 2013). Proyecto 7: "Las condiciones y modos de construcción de subjetividades políticas para la paz en jóvenes de organizaciones interétnicas y feministas desde una perspectiva intergeneracional". Bogotá, Colombia. Recuperado el 18 de 09 de 2015.

Gergen, K. (2011). Reflexiones Sobre el Construccionismo Social. España: Paidós.

Jara, O. (1994). Para sistematizar experiencias: una propuesta teórica y práctica. 3ra edición Alforja. San José, Costa Rica.

Lévinas, E. (1984). Paz y proximidad. Editorial Verdier. Francia.

Lloret, I (2004) Enfoque narrativo y violencia: intervención con mujeres que sufren maltrato. Intervención Psicosocial, 2004, Vol. 13 N. 2 Págs. 165-175 Consultado el 13 de enero de 2015 en: http://www.copmadrid.

Plan de Desarrollo Económico, Social, Ambiental y de Obras Públicas de Facatativá. (22 de enero de 2012). Todos somos Facatativá. Recuperado: 2015 de 10 de 19, de http://www.facatativacundinamarca.gov.co/

Serna, A. (2008). Sentidos y sin sentidos de paz. Bogotá: Universidad Distrital Francisco José de Caldas, Bogotá. Colombia.

Wertsch, J. (1988). Vygotsky y la formación social de la mente. Barcelona: Paidós.

\section{Contenidos}

Al realizar un acercamiento a la experiencia Mujeres Activas del municipio de Facatativá, surgió la pregunta que motivo la investigación: ¿Cómo las prácticas de la ILP de Facatativá: Mujeres Activas, configuran lenguajes de paz?

El trabajo se implementó con el objetivo de acercarse a la población de una manera que permitiera conocer y entender sus percepciones en torno al desarrollo de su participación en la ILP, la construcción social del colectivo de mujeres, sus prácticas potenciadoras de lenguajes y la configuración: lenguajes de paz, estudiados a través de una dimensión situacional, histórica y referencial (Jara, 1994).

En esta medida, la sistematización de experiencias permitió analizar las construcciones históricas y sociales por medio de las cuales se recuperan los saberes y sentidos de las mujeres sobre sus prácticas. Además de ello, uno de los principales aspectos por los cuales se optó por este método, es que al contrario de establecer una marcada diferencia entre sujeto y objeto, y de generar una distancia entre investigadores y participantes, por medio de la sistematización de la experiencia se estableció un proceso de democratización, una relación horizontal y simétrica en la cual no había un sujeto que analizaba y definía las características de un objeto, sino simplemente sujetos que interactuaban y que, partiendo de una situación de interpretación específica, se encontraban y comunicaban. 


\begin{tabular}{|c|c|}
\hline \multicolumn{2}{|c|}{ FORMATO } \\
\hline \multicolumn{2}{|c|}{ RESUMEN ANALÍTICO EN EDUCACIÓN - RAE } \\
\hline \multicolumn{2}{|c|}{ Código: FOR020GIB } \\
\hline Fecha de Aprobación: 10-10-2012 & Persión: 01 \\
\hline
\end{tabular}

\section{Metodología}

Reconociendo la sistematización de experiencias como método, se utilizaron tres técnicas para la investigación:

Los grupos focales se establecieron con la participación de quince mujeres; quienes permitieron el establecimiento de un espacio de interacción, diálogo y participación con el grupo investigador. En éste compartieron relatos sobre la historia colectiva de la experiencia y los aspectos más significativos de la misma, en ellas y su comunidad. En este sentido, se realizaron tres sesiones con los grupos focales dentro de un ámbito de comunicación asertiva que permitieron explicitar sentimientos, sensaciones, creencias y experiencias para poder llegar a entender los verdaderos sentidos y significados de la experiencia, y la manera en que el lenguaje se encuentra instalado como un elemento fundamental para esta iniciativa.

Las entrevistas semiestructuradas se realizaron con las líderes, el psicólogo facilitador Andrés Duarte, el párroco de Santa Beatriz de Silva, Padre Javier Chízica y la facilitadora Alejandra lbáñez, una de las promotoras de pastoral; quienes constituyeron una fuente fundamental para sistematizar la experiencia. Y los talleres a partir del establecimiento de la línea del tiempo de la experiencia, además de brindar información relevante mostrando en sus relatos los sentidos, la historia, y las prácticas desarrolladas por ellas.

\section{Conclusiones}

Mediante su participación, las mujeres han contribuido al desarrollo de procesos de construcción de paz en medio de los cuales se establece un sujeto político femenino, partiendo de la generación solidaria de iniciativas para erradicar la desigualdad de género, y para establecer estrategias que permitan enfrentar efectivamente los problemas que limitan el desarrollo de la comunidad.

Los lenguajes de paz, se hacen presentes en cada actividad de integración en donde las mujeres se reconocen y construyen conocimientos a partir de las experiencias, en cada iniciativa para integrar a la comunidad en la solución de las problemáticas que ellas enfrentan como colectivo, en el desarrollo de una acción social enfocada en garantizar el potenciamiento de las capacidades de la población, y en la generación de una colaboración continua entre ellas, basada en la solidaridad como valor fundamental desde el cual se posibilita la transformación social.

La paz se construye a través de actividades cotidianas, en donde las mujeres pueden dar una nueva interpretación a sus vidas a partir de sus acciones y de su participación. La paz se establece como el camino y al mismo tiempo como la condición que permite mejorar las posibilidades que cada una de ellas tienen para salir adelante y transformar positivamente su entorno, partiendo del uso del lenguaje como una manera de reconocer, de analizar, de reflexionar y de sensibilizar.

La paz no es un concepto abstracto y objetivo, ajeno a las posibilidades reales de la población. Siguiendo a (Serna, 2008) existen tres acepciones para la paz: estado del espíritu, condición social y pretensión política. Por ello es necesario que todos quienes participamos en los ámbitos educativos y del desarrollo social difundamos esta manera de entender la paz, para hacerla constitutiva del mundo personal y del 
plano social; como una forma de poder colectivo que está lejos de ser instalada por la voluntad de unos pocos o por el accionar de un grupo dirigente, sino concerniente a las prácticas que los sujetos promueven en sus contextos particulares.

Dicho de otra manera, la paz se desarrolla en la cotidianidad subjetiva de las personas, en la acción social que determina en gran medida el progreso y el desarrollo humano, en el fortalecimiento de capacidades, en la solidaridad y en la comunicación, en el relato y en la construcción de conocimientos a partir de experiencias, desde las cuales se abre el camino para una paz tal como la promueve el SNPS, que comienza a ser vista como una posibilidad real y no como un ensueño inalcanzable.

\begin{tabular}{|l|l||}
\hline Elaborado por: & Enríquez Duque, Zolangela. \\
\hline Revisado por: & Jiménez Pinzón, Andrea. \\
\hline
\end{tabular}

\begin{tabular}{|l|l|l|l|l||}
\hline $\begin{array}{l}\text { Fecha de elaboración del } \\
\text { Resumen: }\end{array}$ & febrero & 10 & 2016 \\
\hline
\end{tabular}




\title{
Lenguajes de paz: una construcción social femenina Una experiencia de la iniciativa local de paz de Facatativá - Cundinamarca*
}

\author{
Zolángela Enríquez Duque**
}

*Este artículo de investigación científica, presenta avances de la investigación: Las condiciones y modos de construcción de subjetividades políticas para la paz, en jóvenes de organizaciones interétnicas y feministas desde una perspectiva intergeneracional, adelantada por el equipo del que hace parte la autora, del Programa: sentidos y prácticas políticas de niños, niñas y jóvenes en contextos de vulnerabilidad en el Eje cafetero, Antioquia y Bogotá: un camino posible de consolidación de la democracia, la paz y la reconciliación mediante procesos de formación ciudadana, del Consorcio Niños, niñas y jóvenes constructores de Paz: democracia, reconciliación y paz, co-financiado por Colciencias. Código 1235543 31810. Contrato 959 2012. Área Ciencias Sociales. * Fonoaudióloga. Universidad del Rosario. Candidata a Magister en Desarrollo Educativo y Social, Universidad Pedagógica Nacional-CINDE. Docente de la Universidad Cundinamarca. Zolangela_fono@hotmail.com 


\title{
Resumen
}

El presente artículo, como resultado de la investigación sobre las condiciones y modos de construcción de subjetividades políticas para la paz en jóvenes de organizaciones interétnicas $\mathrm{y}$ feministas desde una perspectiva intergeneracional, presenta los resultados parciales relacionados con la sistematización de una Iniciativa Local de Paz ILP, ubicada en el municipio de Facatativá, Cundinamarca, denominada "Mujeres Activas", buscando dar respuesta sobre las prácticas que configuran lenguajes de paz en las integrantes de esta iniciativa.

\begin{abstract}
This article, as the submission of preliminary results for the research, conditions and ways of producing political subjectivities for the peace in young people of interethnic and feminists organizations from an intergenerational perspective, from one of the Local Initiative of Peace - ILP, located in the municipality of Facatativa - Cundinamarca, Called "Mujeres Activas", looking for the answer of the configuration of practices and peace language in the women these initiative.
\end{abstract}

\section{Palabras clave}

Desarrollo Humano, Construccionismo Social, Prácticas, Subjetividad Femenina, Lenguajes de Paz.

\section{Keywords}

Human Development, Social Constructionism, Practices, Female Subjectivity, Peace Languages. 


\section{Tabla de contenido}

Introducción- El problema de investigación - Horizonte metodológico - Construcción social del colectivo de mujeres - Prácticas potenciadoras de lenguajes - Configuración: lenguajes de paz - Conclusiones - Referencias y fuentes.

\section{Introducción}

En este artículo se presentan los resultados parciales de la sistematización de experiencia de la Iniciativa Local de Paz - ILP, denominada Mujeres Activas que se desarrolla en Facatativá - Cundinamarca, en convenio con el Secretariado Nacional de Pastoral Social - SNPS y Ficonpaz. Dicha iniciativa se estudiará desde dos puntos de comprensión.

El primero hace alusión a las prácticas que promueven las mujeres a través de sus procesos de construcción como agrupación. Y el segundo, sobre los sentidos que se establecen alrededor de sus prácticas comunicativas en la manera como configuran lenguajes de paz.

Es importante precisar que los resultados expuestos aquí, aluden tanto a prácticas asociadas con actividades de la Iglesia, como con acciones de carácter social y económico emprendidas por las mujeres, y que dan cuenta de su participación en la iniciativa, en las cuales hacen uso del lenguaje como elemento instituyente para la construcción de paz.

\section{El problema de investigación}

A través del complejo panorama nacional que se presenta en la actualidad, se ha reducido considerablemente el bienestar y las condiciones de calidad de vida de los colombianos. Una de las principales causas que afectan el desarrollo sostenible de las regiones y de la población en el país, es la enorme duración y continuidad del conflicto armado; debido a la interacción de diversos factores, como problemas relacionados con el sector agrario y la repartición de tierras, la limitación que ha existido en el país para 
promover espacios y oportunidades de participación política, la fragmentación institucional y del Estado, además de la ausencia de políticas que garanticen el respeto por los Derechos Humanos y la reparación del tejido social; en donde las personas tengan la oportunidad de desarrollar sus capacidades con el fin de mejorar por sí mismos la calidad de vida de sus comunidades.

Por otro lado, factores como los altos índices de pobreza, la descomposición familiar, la dificultad en muchas regiones del país para acceder a los servicios públicos, además de la falta de oportunidades de formación y empleo, generan un ámbito de inconformidad que muchas veces desemboca en la violencia, donde las principales víctimas han sido las mujeres, niños y jóvenes, que sufren continuamente opresión y discriminación. Por lo cual se hace necesario desarrollar propuestas e iniciativas que fomenten la participación de dicho sector poblacional con el fin de lograr justicia y reparación.

La violencia intrafamiliar y de pareja, son la principal causa de maltrato contra la mujer en nuestro país. El 88 por ciento de los casos reportados se deben a este tipo de agresión. En el año 2012 se presentaron 65.000 casos denunciados y 1.316 homicidios de mujeres víctimas de este tipo de violencia. En conjunto, todo ello hace que las huellas emocionales que deja el abuso en contra de las mujeres sean bastante profundas, lo cual ha complicado en gran medida la tarea de esclarecer las dimensiones y la sistematicidad de este tipo de violencia dentro del contexto nacional. (Consejería Presidencial para la Equidad de la Mujer - CPEM, 2014)

$\mathrm{Al}$ interior del país, en Facatativá - Cundinamarca, las condiciones de vida de las mujeres no escapan a esta realidad. La vulnerabilidad de las mujeres cabeza de hogar y jóvenes se acentúa debido a que no se generan fuentes de empleo suficientes y adecuadas a las necesidades de estas poblaciones. Se presentan frecuentes denuncias por casos de maltrato por parte de sus compañeros permanentes o esposos. Existe desconocimiento por parte de ellas sobre sus derechos, hay permisividad, miedo a vivir sin un hombre que las mantenga producto de criterios de crianza machista. Al interior de las familias, las madres, niñas y abuelas son tratadas de manera diferenciada y desigual frente a los hombres, tanto en el cumplimiento de deberes como en el goce de derechos, debido a 
normas y patrones de tipo cultural; lo que propicia el desconocimiento y omisión de las leyes.

Las instancias gubernamentales que les corresponde brindar asesoría a las mujeres afectadas por la violencia; están permeadas por la misma visión machista, lo que entorpece la garantía del derecho, al realizar la denuncia. Las mujeres de la ILP señalan dentro de este contexto la ineficacia de las rutas de atención al afirmar:

"Las rutas de atención a las denuncias que provienen de la comunidad no son efectivas. Las instituciones no trabajan acorde a las necesidades frente a las denuncias." (Tercer encuentro in-situ- Mujeres Activas, 2013).

Es también notorio que, el número de mujeres que participa en instancias de poder y cargos relevantes en el municipio es mínimo. Existe falta de oportunidades y capacitación, no hay suficientes entidades que se hagan cargo de la atención y educación de los niños y niñas de la primera infancia, por lo que muchas mujeres no pueden realizar actividades diferentes. Un común denominador es la baja autoestima. De otro lado no se tiene en cuenta a la mujer de forma equitativa, subvalorando sus capacidades, conocimientos y generando en algunos casos competencia desleal en el ámbito laboral (Plan de Desarrollo Social, Ambiental y de Obras Públicas de Facatativá, 2012)

En esta medida, a través de las iniciativas desarrolladas para fortalecer y potenciar la participación de la mujer se deben llevar al ámbito público las agresiones y discriminaciones que sufren, a través de espacios de diálogo en donde puedan escuchar y ser escuchadas, con el fin no sólo de compartir sus experiencias, sino también de posicionarse como sujetos políticos. En la medida en que consoliden iniciativas colectivas, proyectos y estrategias de incidencia que les permitan visibilizar sus vivencias, y hacer valer sus derechos, estarán forjando el camino de la construcción de la paz, a partir de sus experiencias para el desarrollo de nuevas capacidades que potencien su lugar como sujeto político femenino. 
Al realizar un acercamiento a la experiencia Mujeres Activas del municipio de Facatativá, surge la pregunta que motiva esta investigación: ¿Cómo las prácticas de la ILP de Facatativá: Mujeres Activas, configuran lenguajes de paz?, con el objetivo de identificar las prácticas que desarrolla el colectivo e interpretar cómo en ellas se establecen lenguajes de paz.

\section{Horizonte metodológico}

El Secretariado Nacional de Pastoral Social (SNPS) es un organismo que surge en el año 1977 para servir en la orientación y promoción del desarrollo integral de la persona a la luz de los valores del Evangelio y del Magisterio de la Iglesia católica. A través del Programa de Construcción de Paz e incidencia, promueve la generación de Iniciativas Locales de Paz en el país. Dentro de ellas, se inscribe la Asociación Mujeres activas, del municipio de Facatativá, Cundinamarca, objeto del presente análisis. De ahí que, mediante un proceso de sistematización de experiencias, interesa hacer visible los diversos modos y condiciones de construcción de subjetividades políticas para la paz y en el caso esta iniciativa, el papel central que juega el lenguaje.

Esta iniciativa se ubica en el barrio la Arboleda, está conformada en su mayoría por mujeres vinculadas a la Diócesis. Participantes de los programas que lidera la parroquia Santa Beatriz de Silva, en evangelización y catequesis. (Secretariado Nacional de Pastoral Social, 2013)

Estas mujeres son en su mayoría cabeza de familia, de estrato socioeconómico bajo, con edades que oscilan entre los 14 y 60 años, cuyo nivel de escolaridad se encuentra entre los grados iniciales de la escuela primaria y algunos años cursados del bachillerato. Hacen parte de grupos familiares con 2 o más hijos, donde la figura masculina no siempre está presente, pues muchas de ellas son separadas o se auto describen como quienes asumen en su totalidad la crianza de los hijos y la manutención de sus familias. 
A partir de las experiencias de este colectivo femenino en contextos de violencia (familiar y social) se desarrollan diversas prácticas, donde se destaca la narrativa como elemento que favorece la construcción de un nuevo tejido social.

El trabajo se implementó con el objetivo de acercarse a la población de una manera que permitiera conocer y entender sus percepciones en torno al desarrollo de su participación en la ILP, este método partió de un criterio dialógico, en donde los fenómenos se estudiaron a través de una dimensión situacional, histórica y referencial (Jara, 1994).

En esta medida, la sistematización de experiencias permitió analizar las construcciones históricas y sociales por medio de las cuales se recuperan los saberes y sentidos de las mujeres sobre sus prácticas. Además de ello, uno de los principales aspectos por los cuales se optó por este método, es que al contrario de establecer una marcada diferencia entre sujeto y objeto, y de generar una distancia entre investigadores y participantes, por medio de la sistematización de la experiencia se estableció un proceso de democratización, una relación horizontal y simétrica en la cual no había un sujeto que analizaba y definía las características de un objeto, sino simplemente sujetos que interactuaban y que, partiendo de una situación de interpretación específica, se encontraban y comunicaban.

Reconociendo la sistematización de experiencias como método, se utilizaron tres técnicas para la investigación:

Los grupos focales se establecieron con la participación de quince mujeres; quienes permitieron el establecimiento de un espacio de interacción, diálogo y participación con el grupo investigador. En éste compartieron relatos sobre la historia colectiva de la experiencia y los aspectos más significativos de la misma, en ellas y su comunidad. En este sentido, se realizaron tres sesiones con los grupos focales dentro de un ámbito de comunicación asertiva que permitieron explicitar sentimientos, sensaciones, creencias y experiencias para poder llegar a entender los verdaderos sentidos y significados de la 
experiencia, y la manera en que el lenguaje se encuentra instalado como un elemento fundamental para esta iniciativa.

Las entrevistas semiestructuradas se realizaron con las líderes, el psicólogo facilitador Andrés Duarte, el párroco de Santa Beatriz de Silva, Padre Javier Chízica y la facilitadora Alejandra Ibáñez, una de las promotoras de pastoral; quienes constituyeron una fuente fundamental para sistematizar la experiencia.

Y los talleres a partir del establecimiento de la línea del tiempo de la experiencia, además de brindar información relevante mostrando en sus relatos los sentidos, la historia, y las prácticas desarrolladas por ellas.

\section{Resultados}

Tomando como base los procesos anteriormente enunciados y con la finalidad de identificar y analizar las prácticas y el establecimiento de lenguajes de paz, desarrolladas por las mujeres de la ILP, objeto del presente estudio, se realiza la presentación de los hallazgos en tres momentos: Construccionismo social, las prácticas de la ILP y las prácticas que configuran lenguajes de paz.

\section{Construcción social del colectivo de mujeres}

Berger y Luckmann, (1996) refieren que "por medio de la interacción entre las personas, de la forma en que se comunican, es posible reproducir una información en realidades sociales donde se establece una realidad compartida.” (p. 128)

De lo anterior se infiere que la realidad no es una verdad absoluta que se establece de manera objetiva, sino que es el producto de la participación de las personas en comunidades de significado, en donde se establecen sentidos compartidos de acuerdo al rol social que cada quien posea. Mediante el establecimiento de tramas de 
conversaciones el sujeto negocia y ratifica de cierta manera su rol, en una relación constante de interdependencia.

Esta manera de construir, interpretar y darle significado a la realidad se hace manifiesta en las palabras de una de las mujeres participantes, al explicar algunas de las actividades que realizaban en grupo:

(...) Ya está conformado el grupo, porque buscamos el nombre el grupo de Mujeres Activas de la Arboleda, en la parroquia del padre... Todas ya empezamos a decir: Bueno, esto es de Pastoral social, de la iniciativas locales de paz que las patrocinan Caritas...Entonces aquí ya hay un rubro, entonces vamos a comprar cosas... ¿qué les gustaría trabajar?... Entonces qué papel reciclable, country, entonces ahí ya se hizo el desembolso...Se sostuvo en esos espacios de escucha de fortalecimiento, de integración, de exponer, de investigar que era no hago nada en mi casa, el tema para el próximo viernes, quienes van a trabajar, decía el grupo... Estudiábamos, investigábamos, veníamos, se exponía, se hacían juegos, fue algo muy enriquecedor para cada una. (María Consuelo Rojas, grupo focal, noviembre de 2013)

A través del desarrollo de la cotidianidad, las personas que comúnmente se relacionan entre sí establecen un tejido conceptual, por medio del cual definen los parámetros que ordenan y le dan sentido a la realidad, y al mismo tiempo establecen los medios por los cuales se puede modificar la acción social. El tejido conceptual se desarrolla a partir de las costumbres de las personas, de sus ritos y maneras de explicar la realidad, convirtiéndose además en la manera fundamental de construir la subjetividad, la identidad y la acción política frente al otro (Domínguez y Herrera, 2013).

Siguiendo a Domínguez y Herrera (2013), refieren que un elemento fundamental del construccionismo social es la edificación de significados mediante el uso del lenguaje. La necesidad de comunicarse y el deseo de recibir información son innatos en el ser humano. Los significados se construyen de manera convenida con otros, ya sean estos reales o imaginarios. De este modo, las narrativas no recrean, crean y obtienen 
significado en el interior de las personas, sino en las relaciones que se establecen con los demás.

La comunicación asertiva sólo se logra por medio de un mensaje relatado, el cual es captado por sus sentidos y depurado a través de las estructuras de pensamiento del individuo. El mensaje es narrado e interpretado por personas que comparten una misma realidad, permitiéndoles de manera conjunta construir sentidos y significados ante los eventos y situaciones que experimentan en la cotidianidad. Todo relato es mezclado con los relatos de los otros, de manera que cada persona se vuelve un actor protagónico en las historias que se cuentan sobre el entorno, el pasado y las experiencias vividas.

Para Lloret (2004), la comprensión de la subjetividad no puede ser separada de la forma en que las personas son narradas, de manera que la comunicación, el diálogo y los espacios de participación se establecen como un aspecto central para el construccionismo social.

Precisamente, en la ILP se han fomentado estos espacios de participación, con el fin de que las mujeres compartan sus experiencias, narren las experiencias que han marcado sus vidas, y a partir de allí puedan construir reflexiones conjuntas desde las cuales se promueven iniciativas y proyectos que les permitan cambiar la concepción que tienen sobre lo que significa ser mujer, por medio de su acción en el hogar, la comunidad y en el mundo.

Teniendo en cuenta lo anterior, es posible entender en qué medida las mujeres de la ILP de Facatativá se auto-producen socialmente, a través de sus prácticas y de su participación, generando una nueva manera de verse y de valorarse a sí mismas, por medio de un elemento fundamental como lo es el lenguaje. Según las palabras de una de las mujeres participantes:

"Transmitimos nuestro conocimiento a otras señoras, a otras mamás o niños que también están viviendo lo de violencia intrafamiliar.... Sí. Nos buscan, para hablar y saber cómo se llama la red de apoyo para decirle a otras señoras...Nosotras les decimos: Vaya a la 
comisaria de familia, a la casa de la mujer... depende. Si necesitan una asesoría de abogado por ejemplo, que vayan a Uniagraria que ahí está la Doctora, que si necesitan algo espiritual le pedimos al Padre si por favor las puede escuchar, como ellos tienen su tiempo también ocupado, nosotros venimos hacer esos, como la red, para que ellos nos ayuden para donde encaminarlos, qué puertas pueden tocar, dónde pueden recibir esas orientaciones pero siempre enfocado a lo social, en lo social a mantener lo familiar." (Gloria Amparo Leyton, grupo focal, abril de 2013)

Esta transmisión de conocimientos es precisamente a lo que apunta el construccionismo social. A través de su participación en la ILP, las mujeres tienen la posibilidad de aprender y de compartir lo aprendido, por medio de procesos de socialización en donde el lenguaje permite no sólo transmitir los significados, sino también establecer una comunicación basada en la comprensión, el respeto y la solidaridad.

El construccionismo social se evidencia en la medida en que las mujeres tienen la posibilidad de retomar los aspectos claves de su cotidianidad, de sus vivencias y sus experiencias, con el fin de comunicarlas a las demás, trasladándolas de la esfera privada a la pública, generando reflexiones y transmitiendo conocimientos desde los cuales se establece una nueva forma de significar la realidad y apropiarse de las decisiones e iniciativas que les permitirán mejorar su bienestar personal, el del entorno y la comunidad que las rodea.

Sin duda alguna, la posibilidad que tienen las mujeres de reinterpretar su realidad y de valorarse a sí mismas de una manera distinta, depende del enfoque que ha tenido la ILP de fomentar el desarrollo humano en la comunidad, a través del fortalecimiento de las capacidades y habilidades que les permita a las personas mejorar sus condiciones, partiendo de su propio esfuerzo y de la acción conjunta e integral de todas las participantes.

Al participar dentro de un grupo como la ILP Mujeres Activas, se les permite a ellas explorar sus miedos, identificar sus intereses, plasmar sus proyectos y construir un sueño. A través de las actividades, las mujeres se reconocen como personas que tienen la 
posibilidad para ejercer liderazgo dentro y fuera del grupo, plantearse metas personales y comunes, para proyectar nuevos horizontes de vida. Ellas encuentran en las acciones del grupo, una oportunidad para comunicar, entablar diálogos que generan acuerdos que optimizan la convivencia, y el discernimiento de las problemáticas en busca de caminos posibles para su solución.

Las propuestas que potencian el desarrollo humano, explica Gergen (2007) le dan voz a la experiencia humana que no ha sido tenida en cuenta o que ha sido silenciada, con el fin de visibilizar las potencialidades individuales y colectivas, como fundamento básico hacia la construcción de relaciones que potencien el bienestar social.

En la ILP, por medio de las actividades se construyen nuevos universos de sentido, a partir de un proceso en donde se generan reflexiones sobre las historias de vida, y se analizan cuáles son los sentidos de su nuevo lugar como sujetos en construcción.

\section{Prácticas potenciadoras de lenguajes}

Para Arendt (1997), la acción es la actividad política por excelencia. Es la praxis mediante la cual los hombres pueden transformar el mundo de la vida política. Para ello, se requiere: Participación de la comunidad, existencia de un espacio público que permite obrar, expresar, deliberar y con palabra y acto insertarse en el mundo humano (pag.206).

Según esta autora, el ser humano tiene la potencialidad de desarrollarse como ser político, en la medida en que configura su acción en el entre nos y se hace visible en el ámbito público. Allí, la comunicación establecida mediante el discurso, toma un papel relevante al posibilitar la deliberación, el disenso, el llegar a acuerdos, lo que a su vez favorece la realización y o afirmación de cambios en el individuo y en el colectivo.

Es valioso detenerse en la observación de las acciones que las mujeres de la ILP han ido realizando como grupo, puesto que han logrado posicionar a la iniciativa como un agente de cambio que promueve y visibiliza diversas problemáticas, en los ámbitos personal, social y familiar, donde se destaca la violencia; esta acción colectiva les ha permitido 
ampliar la conciencia en las mujeres y sus familias, en términos de respeto, reparación y acuerdos para establecer verdaderas dinámicas de paz.

...(...) No es dentro de la misa, sino que cogimos en esa hora la gente que viene más. Según la cantidad de personas les colocamos una carita feliz. Nosotros tenemos planeado que una de nosotras se coloque un amuleto, bueno algo que sea de sensibilización. Con Juliana y el profesor de los monaguillos, nos va a ayudar a hacer una dramatización con los niños, también el padre sabe y él nos va a colaborar en el transcurso de la misa. Se va hacer la dramatización y después de la salida de la misa todas nosotras estaremos repartiendo kits que invitan a las mujeres, aunque todavía no se ha definido si es una mochilita. Está todavía indeciso, en ese kit va una jeringa con un mensaje y va con dulcecitos, va un probador de esos pequeñitos y va con un mensajito, van las venditas, las curitas con un mensajito y a los hombres se les entregará un botón, y pues afuera se va hacer un pendón grande donde habrá un mensaje para que las personas que vayan saliendo lo lean, y escriban lo que piensan. (Ana Ávila, grupo focal, Noviembre 2013)

Arendt (2005), afirma que la acción vivida y narrada no significa nada si no hay quien juzgue lo que se dice y hace, si no afecta a alguien, si no transforman las condiciones o las tramas de relación y poder de alguien, si no es apropiada y recordada por una comunidad plural o comunidad pública.

La afirmación de Arendt tiene sustento en las acciones de las integrantes de la ILP, orientadas a la búsqueda de la transformación de su comunidad al relatar, cómo a partir de su iniciativa las personas habitantes de la comunidad, quienes anteriormente no interactuaban, gracias a la intervención de las mujeres activas, experimentaron un despertar de conciencia frente a la realidad del otro, de su vecina, del habitante de su propio entorno; posibilitando así, la construcción de un tejido social, favorecido entre otras, por prácticas de carácter religioso, promovidas por la Parroquia.

(...) Invitamos a la gente, que saliera de las casas, para que se encontraran con los vecinos y así se fueron haciendo las cosas. De las experiencias de colocar coordinadores en cada zona para empezar hacer eventos significativos cada mes. Entonces de pronto un día le vamos hacer la novena al Señor de los Milagros, invitamos a todas la gente de esa zona. 
Entonces aquí hay dos zonas que son muy fuertes que son la del San Miguel Arcángel y la zona de San Juan Apóstol y las otras personas que se han ido vinculado son de otras zonas... (Gloria Amparo Leyton, grupo focal, abril de 2013).

Dentro de las acciones desarrolladas por las mujeres, se hace evidente su participación en la comunidad, como agentes activos de transformación social al organizar y hacerse participes de acciones de formación en los ámbitos espiritual, personal y de emprendimiento.

Con referencia a la formación espiritual, la participación de las mujeres se manifiesta en las actividades de la parroquia, tales como lecturas dentro de las eucaristías, preparación de los niños para los sacramentos y como puente entre otras mujeres y el sacerdote para realizar apoyo religioso o vocacional a otras personas de la comunidad.

...(...) Nosotras le colaboramos al padre con lecturas o cosas así, cuando se inició el grupo ahí iniciamos como las personas que estaban alrededor del lado del padre. Y gente que después entró, Claudia ya después quería pertenecer al grupo, ya después era catequista, es como esos espacios y por ejemplo a Consuelo y a mí, no sé Amparito, nos buscan y nos dicen bueno es que me están dando tal cosa, es que tengo tal problema a dónde puedo ir. Ya la gente nos reconoce y nos busca, y nosotras hacemos el contacto con el padre para que tengan una asesoría espiritual. (María Consuelo Rojas, grupo focal, noviembre de 2013)

Una característica dentro de las acciones que desarrollan las mujeres, es la realización de talleres de formación personal: Comunicación, autoestima, prevención de violencia intrafamiliar entre muchos otros. Lo que ha favorecido el establecimiento de relaciones más sanas en el ámbito familiar y social llegando a generar un cambio significativo en su forma de pensar, sentir y actuar como lo refiere una de las integrantes:

...(...) En el caso de nosotras tuvimos a una compañera, que ella le tenía demasiado miedo al esposo. Estos encuentros le sirvieron a ella para salir de ese miedo que ella tenía y hablar, contar lo que le estaba pasando porque en los talleres que teníamos de valoración, de autoestima todo eso le ayudó a ella a enfrentar el problema que estaba viviendo con el 
esposo, como con sus hijos y salió adelante y ya estudio y está ahorita bien. (Ana Ávila, grupo focal, abril de 2014)

Arendt (2007) plantea que las prácticas de formación se relacionan con la posibilidad que tienen las mujeres de hacerse visibles, pasando de una esfera privada y doméstica a una esfera pública. Dichas prácticas son descritas por las mujeres como espacios de gran riqueza porque les permite develar aspectos de sí mismas, que no habían visto y encontrarse con las otras integrantes del grupo, para intercambiar saberes, experiencias que son transmitidos también a sus familias. Al respecto se comentó:

...(...) Empezando a vincular el tema de estudio, el tema de seguridad personal empezó a afianzarse, con el tema de expresión, algunas familias tienen un componente disfuncional entonces con un ejercicio que se hizo para la familia un sábado, se convocó a las familias para hacer el ejercicio y eso permitió que una de las señoras se pudiera reconciliar con su hija, digamos que eso dio pie para esto. Hay algo que se llama la matriz de la transformación social. (Entrevista, psicólogo Andrés Duarte, febrero 2014)

Las prácticas de formación técnica, como el trabajo de artes y manualidades son señalados por las mujeres de manera significativa, por ser un espacio que las cita para dialogar, para instruirse, capacitarse e integrar a la comunidad alrededor de un proyecto específico. En palabras de Monseñor Héctor Fabio Henao:

(...) Ellas fabrican cosas, hacen arte convierten esas artesanías en productos y comienzan a recuperar entonces la dinámica de la comunidad, se convierten como una levadura, en esa levadura que es fermento en la masa, en esa levadura que hace que la masa se convierta en el pan. (Monseñor Héctor Fabio Henao, video Gente Solidaria: Mujeres Activas).

Arendt (1997) afirma que, la acción política se nutre de historias que conservan la memoria de sus agentes, y que la libertad es su rasgo esencial, pues gracias a ella los seres humanos pueden renovar el mundo mediante sus nuevos proyectos. 
Frente al desarrollo de la acción política de las mujeres de la iniciativa se puede establecer que mediante sus prácticas este grupo se ha visibilizado en el contexto local, lo que les ha facilitado llegar a tener nuevas oportunidades de aprendizaje y experiencias al explorar contextos y espacios inesperados, como es el caso de las invitaciones para participar en ferias empresariales, campañas de prevención de violencia intrafamiliar, cursos en temas de legislación familiar y la realización de un programa de televisión con la programadora Cristovisión. Experiencias que permiten identificar la manera en que la acción de la agrupación las ha hecho visibles como agentes políticos y de cambio en su ámbito social al integrar acciones comunitarias, teniendo como excusa la productividad.

(...) Desde el primer momento se nos pidió buscar algo productivo, pero hoy en día se nos dice que hay que hacer algo comunitario, entonces eso ha generado un tránsito entre lo productivo y lo comunitario. También lo productivo se volvió un espacio de esparcimiento, donde aprendimos a elaborar artesanía en madera, a hacer papel reciclado y ahorita estamos trabajando todo el tema de tarjetería con el papel reciclado y también sacando el tema del country para comercializar. (Ana Ávila, grupo focal, noviembre 2013)

\section{Configuración: Lenguajes de paz}

$\mathrm{Al}$ comienzo de la iniciativa se desarrollaron convocatorias para las mujeres del barrio La Arboleda, con el fin de generar encuentros en donde se analizara el papel de la mujer en la familia y en la comunidad, en sus diferentes facetas, y en donde se desarrollaron distintos tipo de actividades que las integraban, permitiendo generar relaciones de confianza para que pudieran contar y resaltar sus experiencias. Según las palabras del psicólogo fundador de la iniciativa:

(...)...Lo que se quiso fue abrir espacios, por ejemplo donde teníamos teatro, donde hicimos aeróbicos, donde hicimos danzas, donde se empezó a crear acciones conjuntas para la comunidad como celebraciones, el día de la mujer y el día de la madre. Los viernes de 7 a 9 de la noche era el espacio en donde podían asistir. Como cosas interesantes son los resultados que podemos ver: algunas empezaron a estudiar y a terminar sus estudios, porque sentían también el apoyo dentro del mismo grupo. El tema de estudio, el tema de 
seguridad personal empezó a afianzarse... (Entrevista, psicólogo Andrés Duarte, febrero 2014)

Desde el comienzo, la formación y el desarrollo de las capacidades de las participantes se establecieron como aspectos fundamentales de la iniciativa. Inicialmente, mejorar sus habilidades para expresarse, para comunicar sus ideas y compartir sus experiencias con el grupo resultaba vital, para fortalecer la relación entre ellas y para que todas tuvieran la posibilidad de participar activamente en los encuentros:

(...) Creo que fue muy importante el tema de fortalecimiento de habilidades comunicativas que se hizo con ellas a través del teatro. Se trajo un profesor de teatro, donde se realizó proyección de voz, postura frente al escenario, y al lado también estaba la vida de la parroquia que se fortalecía en términos de liderazgo. Entonces algunas ya son lectoras, tienen un papel fuerte en la parroquia y eso ayudó a fortalecer esa parte de seguridad y expresión, frente a ese lenguaje... (Entrevista, psicólogo Andrés Duarte, febrero 2014)

Es notable, la importancia que ha tenido en la iniciativa el desarrollo de una relación de confianza y respeto entre las participantes. Por otro lado, el hecho de que desde el comienzo las actividades se hayan enfocado en fortalecer el diálogo, la expresión y la comunicación, es prueba de que la ILP es, ante todo, un espacio de interacción intersubjetiva, en donde el conocimiento se produce a partir de la interacción y del desarrollo de ideas conjuntas que mejoren la calidad y el bienestar de vida, tanto de las mujeres participantes como de la comunidad.

En este sentido, es importante analizar la relación que existe entre el concepto de lenguajes de paz con el de intersubjetividad, el cual establece una relación activa entre las personas, por medio de un intercambio en donde suceden diferentes experiencias y acciones, que permiten trasladar las ideas y los conocimientos de la esfera privada a la esfera pública. Para Rogoff (1993), la intersubjetividad hace referencia al proceso de comunicación verbal y no verbal, y se entiende como una actividad social en donde se establece un puente entre dos interpretaciones de una misma situación. 
Por lo tanto, la intersubjetividad, vista como aquello que les permite a las personas generar significaciones y sentidos compartidos, ha sido una condición clave para el desarrollo de los lenguajes de paz, en la medida en que este tipo de construcciones sociales sólo pueden hacerse efectivas en medio de la implementación de espacios de diálogo, reflexión y comunicación, en donde de manera conjunta sea posible llegar a acuerdos sobre un fenómeno o una situación específica.

En este punto, cabe resaltar las consideraciones de Wertsch (1988) quien plantea que la intersubjetividad se genera cuando los interlocutores comparten algún aspecto de sus significaciones sobre una situación. Por lo tanto, la intersubjetividad se construye en el marco de la acción y de la actividad que realizan conjuntamente las personas, que permite la posibilidad de elaborar significados compartidos.

Las mujeres participantes comparten una serie de situaciones y experiencias comunes que las han llevado a identificarse unas con otras, y a establecer unas pautas de comunicación conjuntas, mediante las cuales se interpretan los significados de acuerdo a aquello que tienen en común. Las mujeres de la iniciativa, a partir de sus relatos, construyen una nueva subjetividad, empleando un lenguaje que fomenta la construcción colectiva de acciones de paz, como la valoración de las historias de las otras mujeres, la colaboración, el respeto y la solidaridad.

\footnotetext{
"Con todo lo que viene...los talleres de origami, con los talleres de papel... todo eso enriquece, pero el grupo no ha girado alrededor de eso... de hacer cosas. No. Sino, lo que decía: a ser tolerantes, que es la parte humana y el secreto de esta experiencia." (Ana Ávila, grupo focal, noviembre 2013)
}

A partir del diálogo, de la reflexión en torno a los relatos y experiencias de las mujeres, se produce un lenguaje de paz en dos sentidos distintos: en primer lugar, porque los espacios en donde se narran y analizan las experiencias parten de la base de la solidaridad, el respeto y la comprensión; y en segundo lugar, porque a partir de la reflexión se generan acciones, iniciativas y propuestas en donde se involucra la participación de la comunidad, con el fin de visibilizar las problemáticas, de hacer notar 
en la esfera pública las crisis que debían enfrentar las mujeres en su privacidad, y de establecer soluciones efectivas que permitan generar un mejor bienestar y un mayor desarrollo humano.

En este contexto, la "paz" no proviene de un concepto aislado del devenir y de la acción humana, sino de los acontecimientos y de las transformaciones que se generan por los individuos en su participación social. En el sentido expresado por Lévinas (1984), la paz es un primer lenguaje, pensado a partir de la verdad que en lugar de oponerse, se acuerda o se une. (pág. 339)

La construcción de la paz, por lo tanto, se desarrolló entre las mujeres que participan en la ILP como una aptitud a la palabra, como una acción que consiste en narrarse a través de sus experiencias, desenterrarlas del pasado, hacerlas presentes, compartirlas y superarlas. De esta forma, la construcción de la paz equivale, no en menor medida, a un proceso de reconciliación en donde las mujeres aprenden a aceptar lo pasado y a desarrollar las transformaciones que sean necesarias para no volver a vivirlo, y para que, haciéndose responsables de sus propias acciones, como sujetos políticos que se configuran en la comunidad, fortalecen el conjunto de capacidades que les permitirán alcanzar la justicia, la tranquilidad y la paz.

En la ILP, la paz se construye a través de actividades cotidianas, como la preparación de las eucaristías, las lecturas en las misas, la adquisición de materiales para los talleres, la preparación de las actividades para la catequesis, o simplemente el encuentro con los vecinos en el barrio, generando espacios para el encuentro e integración de la comunidad, para sensibilizar a las personas en torno a la importancia de erradicar la violencia y la opresión, y en el desarrollo continuo de habilidades y capacidades que les permitan enfrentar los retos que se les impone en la cotidianidad, mejorando las posibilidades que tienen para salir adelante y transformar positivamente el concepto que tienen sobre lo que significa ser mujer.

Son precisamente estas nuevas creaciones suscitadas en las mujeres a través de su participación en la ILP las que permiten la construcción de un camino de paz. En palabras de uno de los facilitadores del proyecto: 
(...) Yo si valoro algo del grupo de mujeres activas: Es que ellas no empezaron la experiencia de paz a partir de lo académico, sino a partir de lo humano, eso es fundamental, o sea la familia. Encontrarse con la otra, ser tolerantes, eso son cosas muy humanas... (Entrevista, Párroco Javier Chizica, abril 2014)

Según Lévinas (1984) al hablar de iniciativas y acciones para la paz, se hace necesario impulsar la creación de redes de acción, en donde todos tengan la oportunidad de participar. Por medio de la participación en espacios que promueven el diálogo y la reflexión en torno a una realidad común, se establece un empoderamiento pacifista, entendido como el reconocimiento de capacidades de las prácticas y acciones pacíficas para transformar positivamente el entorno, y para promover la creación de redes entre los actores que se interesan por la paz:

(...) Todo es parte de lo fundamental, piensan que las experiencias de paz están demasiado condicionadas a aprender cosas, no es aprender cosas, es vivir experiencias... Esto es vivir una experiencia. Por eso creo que el éxito de esto es precisamente eso: el compartir con un ser humano y lo enriquecemos con todo lo que viene con talleres de papel, todo eso... Se enriquecen, pero porque el grupo no ha girado alrededor de eso. De hacer cosas no, sino lo que decía... de ser tolerantes, es la parte humana es el secreto de esta experiencia. (Entrevista, Párroco Javier Chízica, abril 2014)

Por lo tanto, la construcción de la paz es, ante todo, una experiencia en donde las personas se comunican y reconocen la importancia de valores como la solidaridad y el respeto, para ponerse en el papel de otro y desde allí formular estrategias viables para remediar las situaciones negativas.

Finalmente, para terminar este análisis, vale la pena citar las palabras del psicólogo promotor de la iniciativa Andrés Duarte, quien expresa:

...(...) El lenguaje de la paz, es un lenguaje de cotidianidad y desde ese punto de vista si siento que hay avances en la medida que se vuelven el apoyo para otras mujeres y familias, en temas como el maltrato. Igual se busca los nexos con la pastoral social o la 
casa de la mujer, para que no se dé más situaciones de maltrato. El tema de formación que se da sobre violencia y no violencia es como un punto de partida de ellas para decidir no más y poderlo transmitir a la comunidad (...) Entonces si siento a las señoras del grupo en un punto de enlace para que aborden a la persona que tienen mayor confianza y con ella se transmite la idea. Entonces siento que su lenguaje, es un lenguaje con hechos no que tanto hablen de paz, paz. Sino que dicen a las demás. ¿Por qué no estudia?, venga salga adelante..., venga no se deje golpear más... ¿̇Por qué no volvió al grupo? ¿Le pasa algo? Es preocuparse por el otro, que uno sienta que su responsabilidad no solo es la que tiene que ver con su núcleo familiar, sino con la de todos los otros. (Entrevista, psicólogo Andrés Duarte, febrero 2014)

\section{Discusión}

Para realizar el análisis que permita establecer cómo se han desarrollado los lenguajes de paz a través de la ILP, ha sido necesario, en primera instancia, determinar cuáles han sido los enfoques de la iniciativa, estableciendo qué capacidades son las que se han pretendido fortalecer en las mujeres participantes, y cuáles son los procesos que se siguieron para alcanzar los objetivos.

Por medio de este análisis se llegó a la conclusión de que la principal capacidad que se quiso fortalecer por medio de las actividades fue la comunicación, a través del diseño de espacios en donde las mujeres aprendían a expresarse, a contar sus historias, narrar sus experiencias y discutir sus ideas. El hecho de que al comienzo la iniciativa haya contado con un profesor de teatro que les enseñaba a las mujeres expresión corporal es prueba de que la comunicación fue un aspecto fundamental en el desarrollo de la ILP en Facatativá.

La creación de espacios de diálogo, análisis y reflexión fue importante en la medida en que permitió a las mujeres contar sus historias, dándoles una nueva significación, para así superar sus experiencias dolorosas y enfocarse en el desarrollo de las capacidades que les permitirán mejorar su situación. El diálogo, además, favoreció el mejoramiento progresivo de una relación de confianza entre las mujeres, para que aprendieran a reconocerse en las demás, para que entendieran la importancia de compartir sus 
vivencias y sus experiencias, y para que pudieran trabajar de manera conjunta, iniciando un proceso de desarrollo humano en medio del cual pudieran reinterpretar el significado de ser mujer.

El diálogo, la comunicación y la expresión se generaron en medio de un ambiente armónico, en donde valores como el respeto y la solidaridad eran la base que permitía el intercambio de experiencias. En el proceso, las mujeres se convertían en cómplices, aprendían a reconocer las problemáticas de las demás y a participar en iniciativas de manera conjunta para integrar a la comunidad, para hacer visibles sus problemáticas y para reducir y eliminar cualquier tipo de opresión o violencia que pudieran haber sufrido en su privacidad.

De esta manera, a través del desarrollo de iniciativas, del intercambio de experiencias, de la participación en proyectos para fortalecer las capacidades, de la acción social y del uso continuo de la narrativa y del relato, se potenciaban los lenguajes de paz, como una condición fundamental para reducir el maltrato y la opresión, y para fortalecer las posibilidades de un desarrollo enfocado en las potencialidades de las mujeres.

Mediante su participación, las mujeres han contribuido al desarrollo de procesos de construcción de paz en medio de los cuales se establece un sujeto político femenino, partiendo de la generación solidaria de iniciativas para erradicar la desigualdad de género, y para establecer estrategias que permitan enfrentar efectivamente los problemas que limitan el desarrollo de la comunidad.

Los lenguajes de paz, se hacen presentes en cada actividad de integración en donde las mujeres se reconocen y construyen conocimientos a partir de las experiencias, en cada iniciativa para integrar a la comunidad en la solución de las problemáticas que ellas enfrentan como colectivo, en el desarrollo de una acción social enfocada en garantizar el potenciamiento de las capacidades de la población, y en la generación de una colaboración continua entre ellas, basada en la solidaridad como valor fundamental desde el cual se posibilita la transformación social. 
La paz se construye a través de actividades cotidianas, en donde las mujeres pueden dar una nueva interpretación a sus vidas a partir de sus acciones y de su participación. La paz se establece como el camino y al mismo tiempo como la condición que permite mejorar las posibilidades que cada una de ellas tienen para salir adelante y transformar positivamente su entorno, partiendo del uso del lenguaje como una manera de reconocer, de analizar, de reflexionar y de sensibilizar.

El principal elemento de discusión que se establece a partir del artículo, es que la paz no es un concepto abstracto y objetivo, ajeno a las posibilidades reales de la población. Siguiendo a (Serna, 2008) existen tres acepciones para la paz: estado del espíritu, condición social y pretensión política. Por ello es necesario que todos quienes participamos en los ámbitos educativos y del desarrollo social difundamos esta manera de entender la paz, para hacerla constitutiva del mundo personal y del plano social; como una forma de poder colectivo que está lejos de ser instalada por la voluntad de unos pocos o por el accionar de un grupo dirigente, sino concerniente a las practicas que los sujetos promueven en sus contextos particulares.

Dicho de otra manera, la paz se desarrolla en la cotidianidad subjetiva de las personas, en la acción social que determina en gran medida el progreso y el desarrollo humano, en el fortalecimiento de capacidades, en la solidaridad y en la comunicación, en el relato y en la construcción de conocimientos a partir de experiencias, desde las cuales se abre el camino para una paz tal como la promueve el SNPS, que comienza a ser vista como una posibilidad real y no como un ensueño inalcanzable. 


\section{Referencias y Fuentes}

Arendt, H. (1997) ¿Qué es la política?, trad. de Rosa Sala Carbón, Barcelona, España. Paidós. Edición póstuma 1993.

Arendt, H. (2005) Ensayos de la comprensión 1930-1954. Barcelona, España. Paidós

Arendt, H. (2007). La condición humana. Barcelona, España. Paidós.

Berger, P, \& Luckmann, T. (1996). La construcción social de la realidad. Madrid, España Editorial Pingüino.

CINDE, Universidad de Manizales, Universidad Pedagógica (26 de Agosto de 2013). Proyecto 7: "Las condiciones y modos de construcción de subjetividades políticas para la paz en jóvenes de organizaciones interétnicas y feministas desde una perspectiva intergeneracional”. Bogotá, Colombia. Recuperado el 18 de o9 de 2015.

Consejería Presidencial para la Equidad de la Mujer - CPEM. (2014). Informe al Congreso 2013-2014. Bogotá.

Domínguez, J y Herrera, E. (2013). La Investigación Narrativa en Psicología. Revista Psicología desde el Caribe. ISSN 2011-7485 (Publicación on line), Vol. 3.

Gergen, K. (1996). Realidades y relaciones: aproximaciones a la construcción social. Meler, F. (Trad.). Barcelona, Buenos Aires: Paidós.

Gergen, K. (2011). Reflexiones Sobre el Construccionismo Social. España: Paidós.

Jara, O. (1994). Para sistematizar experiencias: una propuesta teórica y práctica. 3ra edición Alforja. San José, Costa Rica.

Lévinas, E. (1984). Paz y proximidad. Editorial Verdier. Francia.

Lloret, I (2004) Enfoque narrativo y violencia: intervención con mujeres que sufren maltrato. Intervención Psicosocial, 2004, Vol. 13 N. 2 Págs. 165-175 Consultado el 13 de enero de 2015 en: http://www.copmadrid.

Plan de Desarrollo Económico, Social, Ambiental y de Obras Públicas de Facatativá. (22 de enero de 2012). Todos somos Facatativá. Recuperado: 2015 de 10 de 19, de http://www.facatativa-cundinamarca.gov.co/

Rogoff, B. (1993). Aprendices del pensamiento. El desarrollo cognitivo en el contexto social. Barcelona: Paidós. 
Secretariado Nacional de Pastoral Social. (2013). Estrategia de Intervención. Recuperado el 8 de 10 de 2015, de http://www.new.pastoralsocial.org.

Serna, A. (2008). Sentidos y sin sentidos de paz. Bogotá: Universidad Distrital Francisco José de Caldas, Bogotá. Colombia.

Wertsch, J. (1988). Vygotsky y la formación social de la mente. Barcelona: Paidós.

\section{Fuentes}

Alejandra Ibáñez: Promotora Iniciativa Local de Paz- Facatativá.

María Consuelo Rojas. Promotora Iniciativa Local de Paz - Facatativá

Ana Ávila. Promotora Iniciativa Local de Paz - Facatativá

Gloria Amparo Leyton. Promotora Iniciativa Local de Paz - Facatativá

Andrés Duarte: Psicólogo fundador y promotor.

Padre Javier Chizica: Párroco Iglesia Santa Beatriz de Silva - Facatativá. 